\title{
Solenopora ladinica n. sp. und Solenopora suhadolica n. sp. (Rotalgen) und Paragondolella ? trammeri (Kozur, 1972) (Conodonta) aus dem Ladin (Mitteltrias) bei Suhadole, östlich von Ljubljana, Slowenien
}

\author{
Solenopora ladinica n. sp. in Solenopora suhadolica n. sp. \\ (rdeče alge) in Paragondolella ? trammeri (Kozur, 1972) \\ (Conodonta) iz ladinija (srednji trias) pri Suhadolah, \\ vzhodno od Ljubljane
}

Anton Ramovš

Katedra za geologijo in paleontologijo

Univerza v Ljubljani, Aškerčeva 2, 1000 Ljubljana, Slovenija

Schlüsselworte: Rotalgen, Konodonten, Ladin-Mitteltrias

Ključne besede: rdeče alge, konodonti, ladinij - srednji trias

\section{Zusammenfassung}

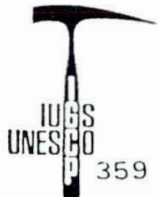

Südwestlich der Ortschaft Suhadole, östlich von Ljubljana, ist ein Riffkalk mit kleinen patch reefs von fächerförmigen, stark mit Kalk inkrustierten Rotalgen Solenopora ladinica n. sp. und Solenopora suhadolica n. sp. und mit kleinen patch reefs von phaceloiden Korallen Tropidendron rhopalifer Cruif, 1957 aufgeschlossen. Die umgebenden schwarzen Plattenkalke führen die Conodontenart Paragondolella? trammeri (Kozur, 1972), die ladinisches Alter (Mitteltrias) des Riffkalkes beweist.

\section{Kratka vsebina}

Južnovzhodno od vasi Suhadole, vzhodno od Ljubljane, je razgaljen grebenski apnenec z majhnimi patch grebeni pahljačastih, močno z apnencem inkrustiranih rdečih alg Solenopora ladinica n. sp. in Solenopora suhadolica n. sp. in faceloidnih koral vrste Tropidendron rhopalifer Cruif, 1957. Opisani sta novi vrsti rdečih alg. $\mathrm{V}$ črnih ploščastih apnencih, ki obdajajo grebene, je ugotovljena konodontna vrsta Paragondolella? trammeri (Kozur, 1972), ki dokazuje ladinijsko starost alginega in koralnega apnenca. 


\section{Einführung}

Südwestlich der Ortschaft Suhadole, östlich von Ljubljana befindet sich am linken Hang des Bučavnica-Baches ein massiger Riffkalk (Abb. 1). Der tektonisch zerklüftete und erosionsdeformierte Riffkalk-Körper erreicht in der Mitte seine grösste Mächtigkeit von $20 \mathrm{~m}$ und verjüngt sich nach beiden Seiten. Am Hang nördlich des Feldweges gibt es nur vereinzelte kleinere Vorkommen eines massigen Kalkes mit der grössten Mächtigkeit von $2 \mathrm{~m}$. Auch nördlich und nordöstlich von der beschriebenen Lokalität sind vereinzelte kleine Riffkalk-Körper aufgeschlossen.

Den mächtigsten Riffkalk-Körper baut ein schwarzer mikritischer bis feinkörniger, mit weissen Kalzitadern durchzogener Kalk auf. Sehr häufig sind kleine Hornstein-Körnchen vorhanden. Im tieferen und oberen Abschnitt des Riffkalk-Körpers sind Büschel von kleinen Korallen-Kolonien charakteristisch. Mehr oder weniger zahlreich sind Echinodermenreste, zarte Kalkschwämme (Inozoa) kommen häufig vor.

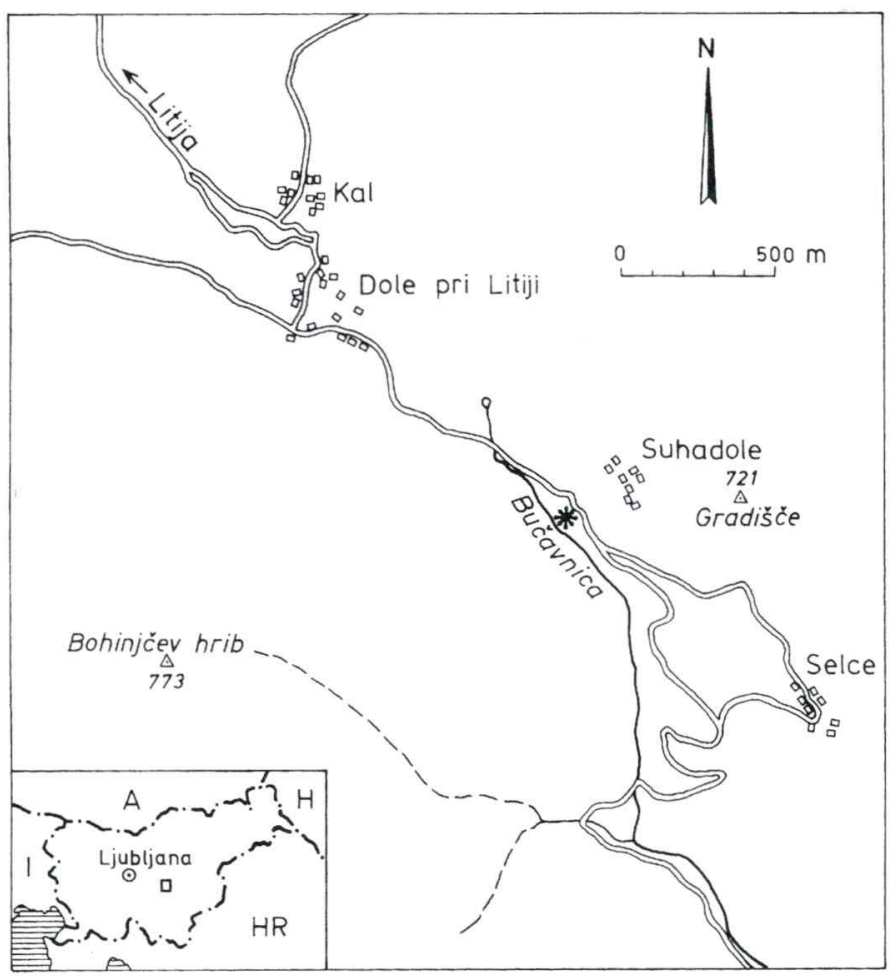

Abb. 1. Die Lage des Fundortes der Rotalgen Solenopora ladinica n. sp. und Solenopora suhadolica n. sp.

Sl. 1. Najdišče rdečih alg Solenopora ladinica n. sp. in Solenopora suhadolica n. sp. 
Im unteren Teil des Riffkalk-Körpers kommen Exemplare der neuen Rotalge Solenopora ladinica $\mathrm{n}$. sp. vor.

Im mittleren und oberen Teil des Riffkalk-Körpers sind innerhalb des mikritischen Kalkes in Segmente geteilte fächerförmige, mit Kalk inkrustierte Thalli der neuen Rotalge Solenopora ladinica n. sp. charakteristisch. Sie bilden bis etwa $25 \mathrm{~cm}$ breite und ungefähr $20 \mathrm{~cm}$ hohe dicht beinander stehende Thalli. In anderen Kalkabschnitten kommen Korallen-Büschel vor, bis etwa $20 \mathrm{~cm}$ lang und etwas weniger hoch. Korallen (Tropidendron rhopalifer Cuif, 1975) sind klein und umkristallisiert. Im Bereich des astförmigen Korallen-patch reefs kommen keine Rotalgen vor und umgekehrt. Äusserst selten treten Einzelkorallen vor, sie haben einen Durchmesser bis $1,5 \mathrm{~cm}$. Schlecht erhaltene unbestimmbare Schalenreste und Echinodermenreste befinden sich in Bereich ohne Korallen und Rotalgen.

Der Riffkalk ist vom schwarzen mikritischen Plattenkalk mit wechsellagernden dünnen mergeligen oder tonigen Lagen umgeben. Diese Kalke führen seltene Conodonten mit der stratigraphisch wichtigsten Art Paragonodolella ? trammeri (Kozur, 1972), die auch das laninische Alter der Riffkalke beweist.

Die neuen Solenoporen- und Korallen-Riffkalk-Körper hat Goce Mitrevski entdeckt und mich auch zu der Fundstelle geführt. Dafür bin ich ihm sehr dankbar.

Bekannte Solenoporen in der Mittel- und Obertrias in Slowenien

Vereinzelte Solenopora sp.-Exemplare im massigen mittelanisischen Kalk am Schlossberg von Bled ähnelten nicht den Solenoporen von Suhadole. Sie bilden auch keine buildups (cf. Flüg el et al., 1994, Taf. 2, Fig. 5-7).

In Slowenien sind meines Wissens in den ladinischen Kalken keine riffbildenden Rotalgen bekannt.

Im karnischen Riff von Hudajužna tritt die Gattung Solenopora nur mit einigen Thalli auf und spielt als Gerüstbildner keine besondere Rolle (Senowbary D a ry a n, 1981, 112).

Die unterkarnische Solenopora sp. von Mežakla, Mojstrana und Vitranc ähnelt im Wachstum der ladinischen Solenopora ladinica von Suhadole, ist jedoch nicht gesteinsbildend und kommt nur vereinzelt vor. Sie hat auch eine viel feinere und einheitliche Struktur. Im Thallus sind bis etwa 45 Zellenlagen (cf. R a m ov š \& Turnš ek, 1984, 183, Taf. 10, Fig. 5). Solenopora alciformis Ott, 1966 vom Zatrep zwischen dem Kot-Tal und dem Vrata-Tal bildet kleine ramose Kolonien und ist mit den Solenoporen von Suhadole nicht vergleichbar (cf. Ramovš \& Turnšek, 1984, 183, Taf. 10, Fig. 6).

Auch Solenopora sp. in den Jul/Tuval-Kalken von Pokljuka ist mit beiden Solenopora-Arten von Suhadole nicht vergleichbar (cf. T u r n š ek \& B u s e r, 1989).

Im Begunjščica-Gebirge, S-Karawanken, sind die Rotalgen durch die Art Solenopora styriaca Flügel, 1960 vertreten. Sie ist in typischer Form mit nodularen Thalli aus perlschnurförmigen Zellfäden entwickelt (Flügel \& Ramovš, 1961, 291). Dieselbe Art ist in den Nor/Riffkalken in den nördlichen Julischen Alpen, am Šplevta-Berg bekannt. Sie kommt nur vereinzelt vor und ist mit Solenoporaceen von Suhadole nicht vergleichbar (cf. Turnšek \& R a movš, 1987, 45, Taf. 15, Fig. 6). 
Zur Bildung der Solenoporen buildups bei Suhadole

Die kleine neue Solenopora ladinica ist nicht in Segmente gegliedert und bildet reguläre, kreisförmig angeordnete Zellenlagen. Sie sind ziemlich hoch. Es ist keine wesentliche Unterbrechung der Zellen-Lagen zu verfolgen. Kleine Hornstein-Körner haben stellenweise den Zellenwuchs verhindert.

Auch bei der wesentlich grösseren, in Segmente gegliederten Solenopora suhadolica ist deutlich, dass die Zellenlagen nur mit kurzen Unterbrechungen gewachsen sind, eine Zellenlage folgte der anderen. Sie variiren in Höhe und Länge. Die charakteristischen paralell angeordneten polygonalen Zellen-Lagen verlaufen im unteren Abschnitt der Segmente ununterbrochen und meist gleichmässig gewölbt. Im verbreiteten mittleren und oberen Teil der Segmente bilden sie meist höhere und niedrigere und untenschiedlich lange Zellenlagen. Gegen die senkrechten oder schrägen Segment-Grenzen keilen die Zellenlagen stumpf oder spitz aus.

In den Solenoporen buildups bei Suhadole kann man keine Wechsellagerung von organischen und sedimentären Lagen beobachten. Der Wuchs von kleinen Solenoporen-patch reefs ist nicht unterbrochen worden. Zeitweise hat sich während des Wuchses mechanische oder biologische Erosion manifestiert. In einigen Segmenten haben Anhäufungen von Mikrofossilien und- oder vom Feindetritus zwischen den Solenoporen-Zellenlagen den Algenwuchs kurz unterbrochen. Bei dem Bau der beschriebenen Solenoporen-buildups haben sich Cyanobacterien als stromatolithenbildende Organismen nicht beteiligt.

Die Solenoporen-Zellenlagen-Struktur ist in meistens weissen und teilweise auch in grauen Teilen der buildups noch deutlich erhalten.

In den jetzigen dunkelgrauen und schwarzen, meist mittleren Teilen der Zellenlagen ist die Zellenstruktur durch diagenetische Vorgänge vernichtet. Die Diagenese hat in vereinzelten Abschnitten manchmal unregelmässig zwei oder mehr Zuwachszonen in Mikrit umgewandelt (unregelmässige schwarze Felder, Abb. 5a, 5b).

Wendt (1993) hat sich eingehend mit "Solenoporacean Stromatolites" beschäftigt. Er beschreibt Collenella sp. A aus dem Oberperm von Djebel Tebaga, Tunesien, und Collenella sp. B aus dem unteren Karn von Denti di Terrarossa, Dolomiten, Italien. Beide stellen wahrscheinlich zwei neue Arten dar, er hat jedoch beide Formen als Collenella sp. A und Collenella sp. B dargestellt.

Wendt $(1993,108,109)$ beschreibt auch die Geschichte der Collenella-Untersuchungen, erstmals von Johns on (1963) beschrieben. Er konnte im Material von der Typus-Region keine Mikrostruktur nachweisen. Er kam jedoch zum Schluss, Collenella guadalupiensis sei ein "Solenoporacean Stromatolit", "in which the algal cell structure is totaly obliterated by diagenesis".

Er schreibt: "Therefore it can be speculated that the rhytmic growth of the solenoporacean stromatolites reflects annual oscillation of muddy influx over periodically re-established algal mats. Newertheless these stromatolites do not fit into a simple model of regularly alternating organic and sedimentary layers (W en d t, 1993, 107).

In den Solenoporen buildups von Suhadole sind keine Stromatoliten-Lagen vorhanden. Es handelt sich nur um Solenoporen buildups.

Mineralogische Untersuchungen der Solenoporen-Lagen brachten folgende Ergebnisse: "x-ray diffraction measurement: only calcite mineral was detected. Aragonite mineral was not proved". 


\section{Systematische Paläontologie}

Rhodophyta Wettstein, 1901

Familie Solenoporaceae Pia, 1927

Unterfamilie Solenoporoidae Maslov, 1935

Gattung Solenopora Dybowsky, 1878

Typische Art Solenopora spongioides Dybowsky, 1878

\section{Solenopora suhadolica n. sp.}

Abb. 2, 3

Derivatio nominis: ladinica, nach dem ladinischen Alter des Fundortes.

Holotypus: Abb. 2. Paläontologische Sammlung des Lehrstuhls für Geologie und Paläontologie der Universität Ljubljana, Katalognummer 5330.

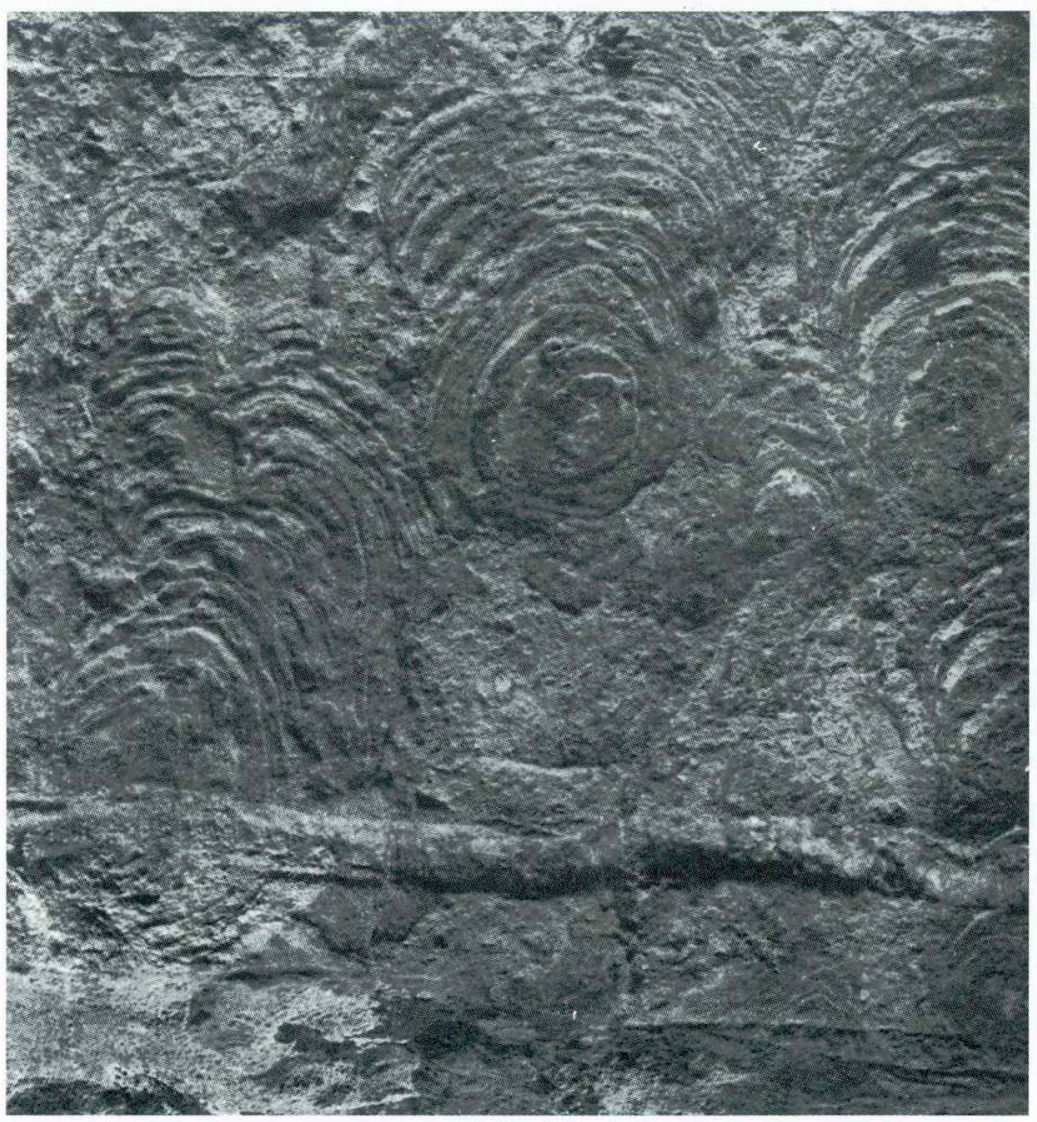

Abb. 2. Solenopora ladinica n. sp. Holotypus. Suhadole, KGP Nr. 5330, Gesteinsstück, Oberfassan, Ladin, Mitteltrias, x 1,5

Sl. 2. Solenopora ladinica n. sp. Holotip. Suhadole, KGP št. 5330, kamninski kos, zgornji fassan, ladinij, srednji trias, $\times 1,5$ 


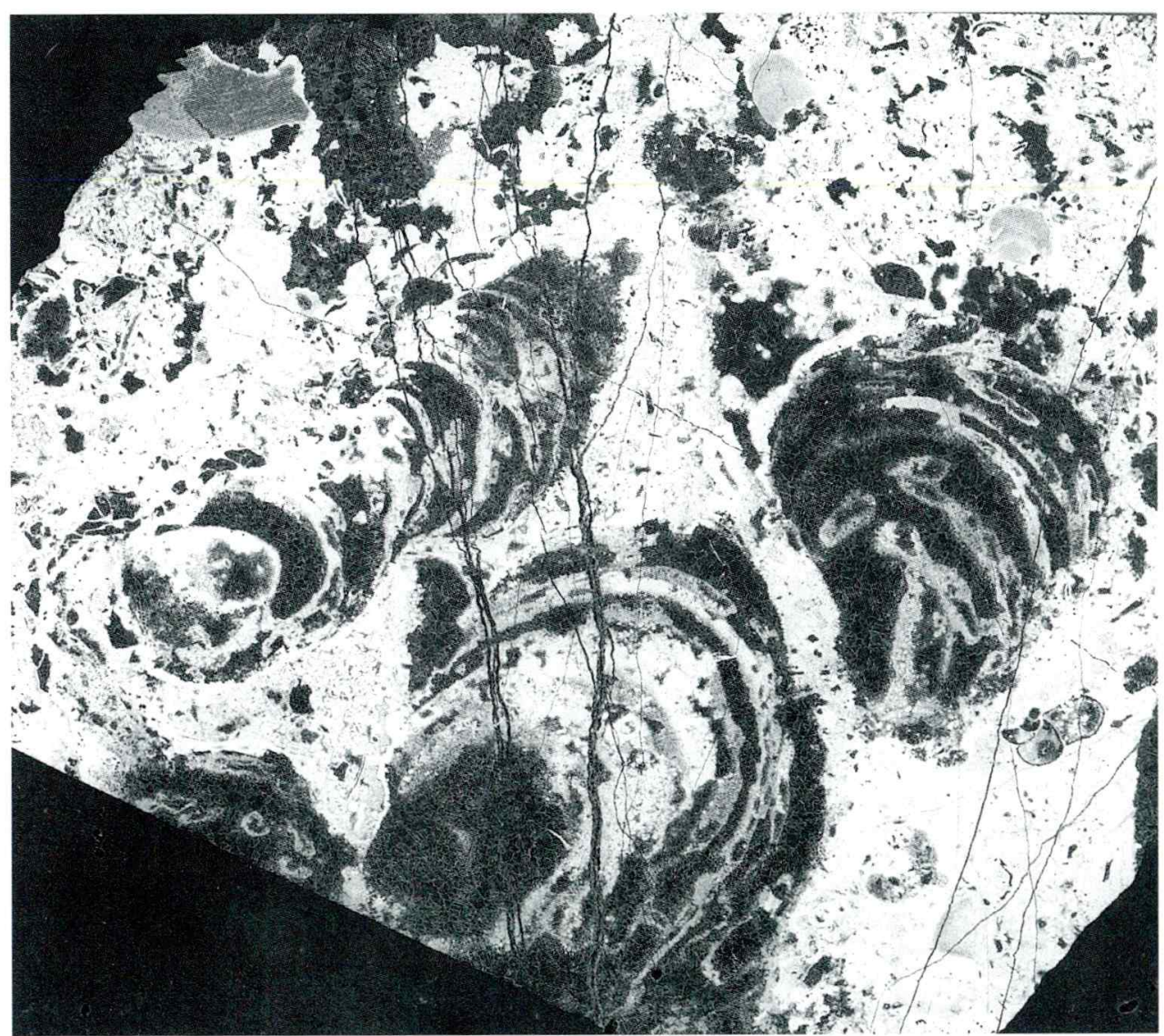

Abb. 3. Solenopora ladinica n. sp. Paratypus. Suhadole, KGP Nr. 5330 a, Dünnschliff, Oberfassan, Ladin, Mitteltrias, $\times 5$

S1. 3. Solenopora ladinica n. sp. Paratip. Suhadole, KGP št. 5330 a, zbrusek, zgornji fassan, ladinij, srednji trias, $\times 5$

Stratum typicum: Riffkalk-Körper südwestlich der Ortschaft Suhadole, östlich von Ljubljana.

Locus typicus: Nordöstlicher Hang des Bučavnica-Baches, südwestlich der Ortschaft Suhadole, östlich von Ljubljana.

Diagnose: Thalus aufrecht, seitlich fächerförmig, nicht segmentiert, mit konvexen konzentrischen Zuwachszonen. Dicht beieinander stehende Zellen sind polygonal. Thalus ist mit Kalk inkrustiert.

Beschreibung: Die Basis des Thallus kreis- bis ellipsenförmig und knollig verdickt. Der Thallus ist bis etwa $3 \mathrm{~cm}$ hoch und $2 \mathrm{~cm}$ breit. Die konvexen Zuwachszonen sind verhältnismässig stark ausgebildet. In den beobachteten Exemplaren kommen auf $3 \mathrm{~cm}$ Höhe 10 bis 11 Zellenlagen vor. Sie sind etwa gleich stark in ganzer Höhe und in regelmässigen Abständen angeordnet. Im angewitterten Zustand sind zwischen den halbkreisförmigen erhöhten Teilen ziemlich tiefe Furchen. 
Beziehungen: Solenopora ladinica stellt eine ältere und einfachere Form der ladinischen Rotalgen im Fundort Suhadole dar. Sie leitet über zu den segmentierten Formen der ladinischen Solenopora, welche auch viel grösser ist. Solenopora ladinica kommt überwiegend im unteren Abschnitt des Riffkalk-Körpers von Suhadole vor. Im höheren Riffkalk-Bereich kommt sie nur noch vereinzelt vor. Im oberen patch reef beobachtet man nur noch grosse, segmentierte Solenopora suhadolica.

Solenopora ladinica unterscheidet sich wesentlich von der Gattung Collenella (Johnson, 1963, 136-137) aus dem Oberperm der Guadalupe Mountains in Nordamerica. Auch mit Collenella sp. A im tunesischen Oberperm und Collenella sp. B im Unterkarn der Dolomiten (W en d t, 1993, Fig. 2 und Fig. 3) ist sie nicht vergleichbar.

\section{Solenopora suhadolica n. sp.}

Abb. 4, 5a, 5b

Derivatio nom in is: nach dem Fundort Suhadole genannt.

Holotypus: Gesteinsstück. Abb. 4, 5a, 5b. Paläontologische Sammlung des Lehrstuhls für Geologie und Paläontologie der Universität Ljubljana. Katalog- Nummer 5331.

Stratum typicum: Riffkalk-Körper südwestlich der Ortschaft Suhadole, östlich von Ljubljana.

Locus typicus: Nordöstlicher Hang des Bučavnica-Baches, südwestlich der Ortschaft Suhadole, östlich von Ljubljana.

D i a gnose: Thallus aufrecht, breit fächerförmig, stark in Segmente gegliedert mit nach oben verschieden breiten, konvexen, konzentrischen, verhältnissmässig schmalen, einander folgenden Zuwachszonen mit polygonalen Zellen. Thallus mit Kalk inkrustiert.

Beschreibung: Die Basis des Thallus kreis- bis ellipsenförmig und knollig verdickt. Der aufrechte Thallus verbreitet sich stark fächerförmig, ist in Segmente geteilt mit leicht konvexen Zuwachszonen. Zwischen den Segmenten verlaufen vertikale oder schräge Furchen. Im Abstand von $4 \mathrm{~cm}$ kommen bis etwa 20 Zellenlagen vor. Sie sind etwa gleich stark bis unterschiedlich hoch und in regelmässigen Abständen angeordnet. Der Thallus kann eine Höhe bis etwa $20 \mathrm{~cm}$ und eine Breite von 25 cm erreichen. Im Thallus treten manchmal kleine schwarze Hornstein-Körnchen auf, die das Wachstum der Zellenlagen verhindert haben.

B ezi e gung en: Solenopora suhadolica unterscheiden sich von Solenopora ladinica durch beträchtliche Grösse, durch einen viel komplizierter gebauten, stark segmentierten Thallus. Solenopora suhadolica hat sich aus der primitiven Solenopora ladinica entwickelt. Solenopora suhadolica zeigt in der Morphologie der Zellen-Lagen und im Zellenbau eine Ähnlichkeit mit der unterladinischen Collenella sp. vom Cipit boulder, Denti di Terrarossa, Dolomiten, Italien (W end t, 1993, 106, Fig. 3). 


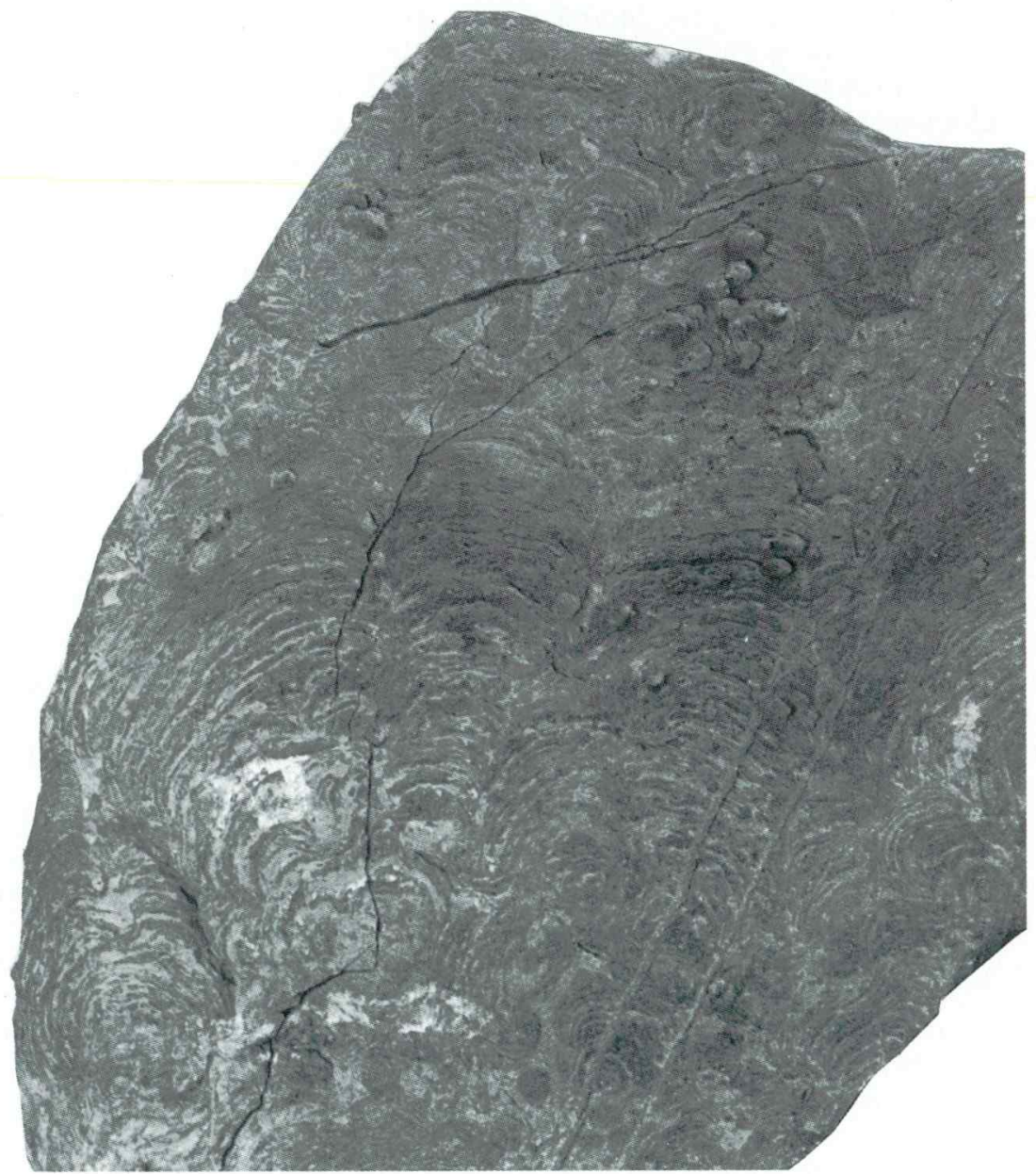

Abb. 4. Solenopora suhadolica n. sp. Holotypus. Suhadole, KGP Nr. 5331, Gesteinsstück, Oberfassan, Ladin, Mitteltrias

S1. 4. Solenopora suhadolica n. sp. Holotip. Suhadole, KGP št. 5331, kamninski kos, zgornji fassan, ladinij, srednji trias

Abb. 5a. Solenopora suhadolica n. sp. Holotypus. Suhadole, KGP Nr. 5331, Dünnschliff vom Holotypus. Vier Segmente mit aneinander folgenden Solenoporen-Zellenlagen. In den unterschiedlich ausgebildeten weissen und hellgrauen Zellenlagen ist die Struktur noch erhalten. In den schwarzen Zellenlagen und in unregelmässigen Partien (Feldern) hat eine feine KalzitKristallisation die Zellenlagen-Struktur umgewandelt. Im ersten und zweiten Segment von links sind in weissen Abschnitten Anhäufungen von Mikrofossilien und Feindetritus, $\times 5$

Sl. 5a. Solenopora suhadolica n. sp. Holotip. Suhadole, KGP Nr. 5331, zbrusek holotipa s štirimi segmenti solenoporskih celičnih plasti. V različno oblikovanih belih in svetlo sivih celičnih plasteh je še ohranjena struktura. V črnih celičnih plasteh in v nepravilnih delih segmentov je kalcitna kristalizacija spremenila algino celično strukturo. V prvem in drugem segmentu od leve so $\mathrm{v}$ belih delih mikrofosili in droben detritus, $\times 5$ 


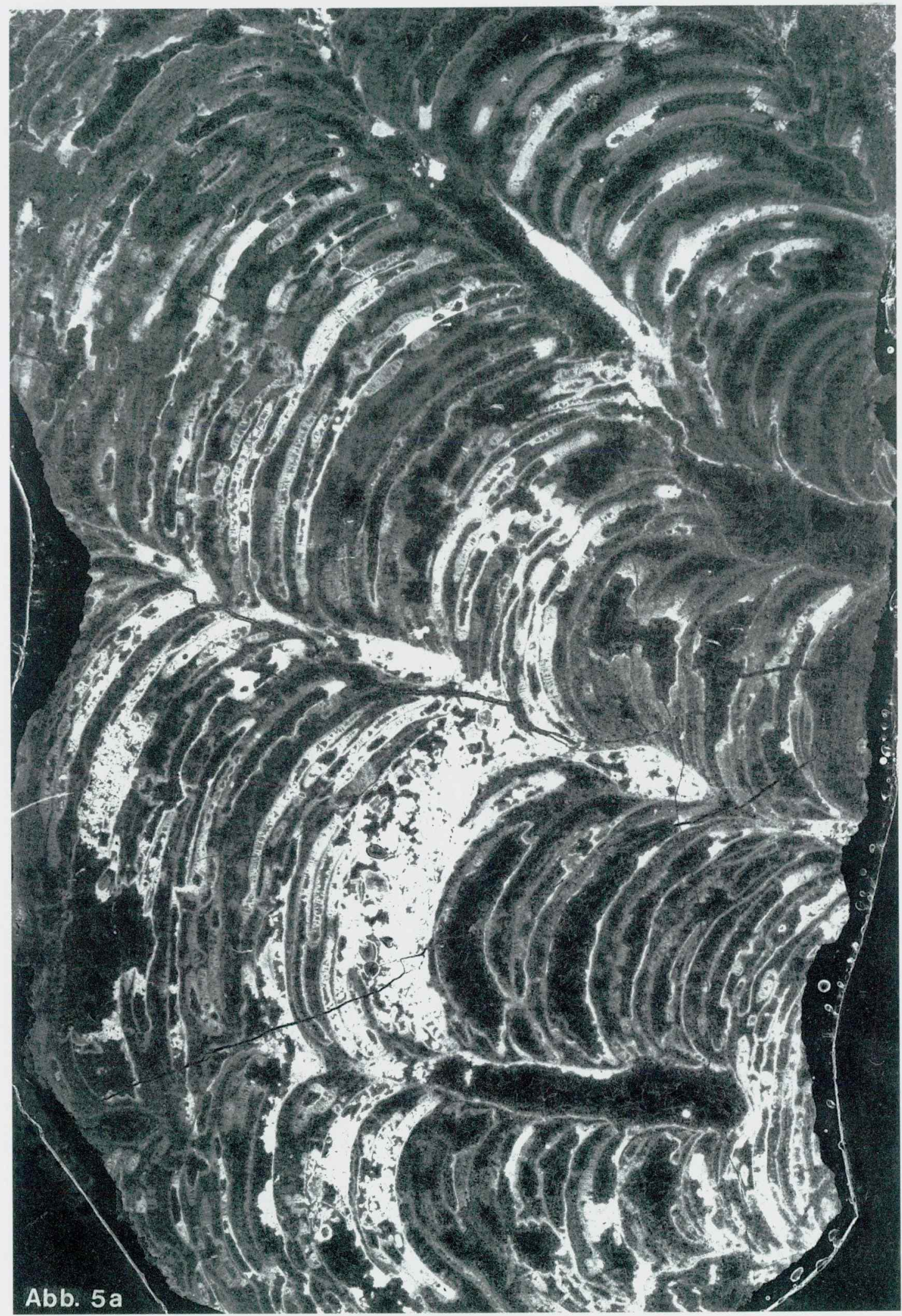




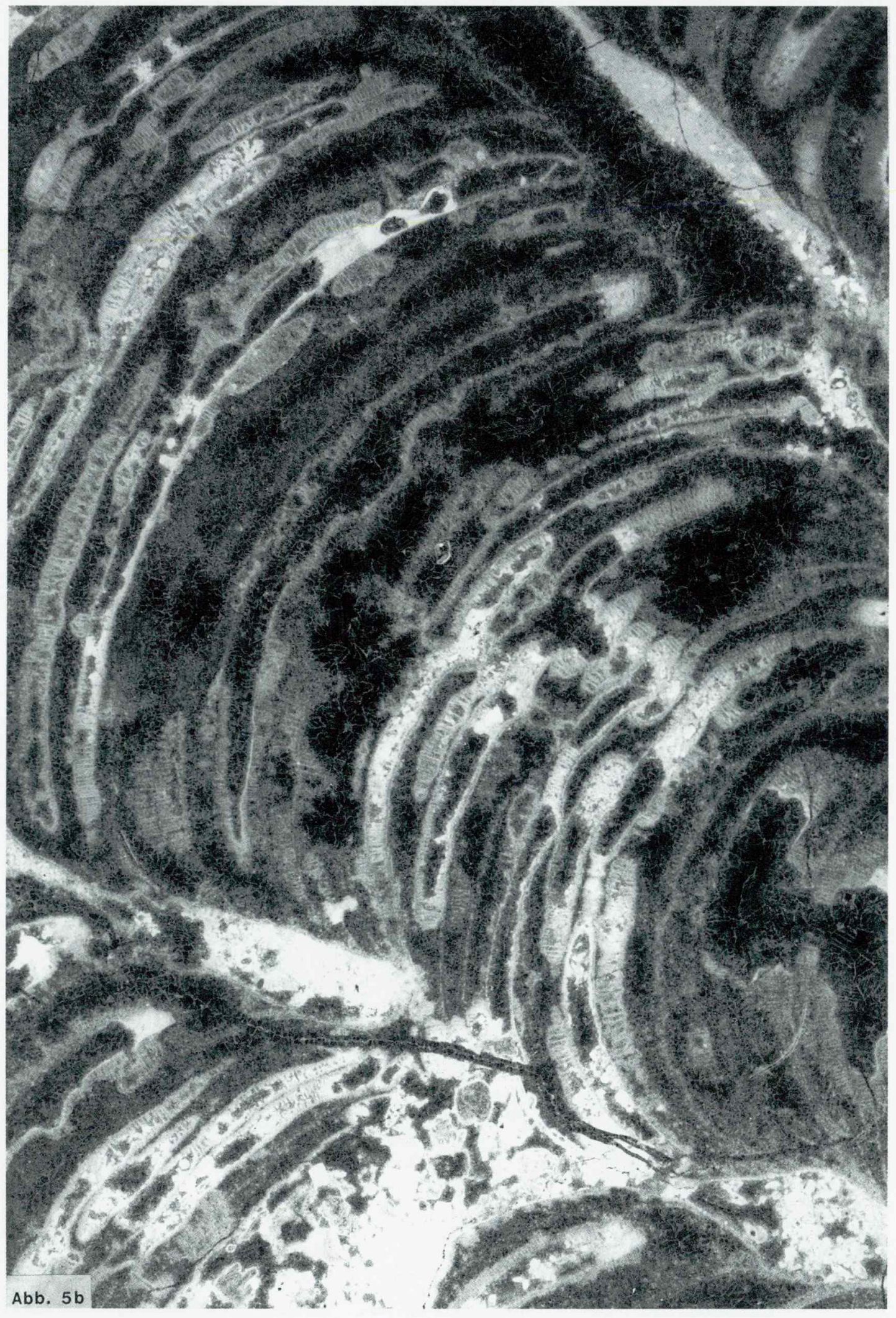


Stamm Conodonta Eichenberg, 1936

Oberfamilie Gondolellacea Lindstöm, 1970

Familie Gondolellidae, Lindström, 1970

Gattung Paragondolella Mosher, 1968

Typusart Paragondolella excelsa Mosher, 1968

Paragondolella? trammeri (Kozur, 1972)

1972 Gondolella haslachensis trammeri Kozur n. subsp. - Kozur und Mock, S. 13, Taf. 1, Fig. 3-5, non Fig. 5, 7.

1980 Gondolella trammeri Kozur emend. - Kovács und Kozur, S. 58, Taf. 6, Fig. 6, 8, non Fig. 7.

1983 Gondolella trammeri Kozur, 1971 (corr. A. R. 1972). - Krystyn, S. 239, Taf. 1, Fig. 5, Taf. 2, Fig. 5-6, Taf. 3, Fig. 3, 4.

Materia l: Zwei Exemplare.

Original-Diagnose: Kozur und Mock, 1972, S. 13.

Beschreibung: Ramovš, 1994.

Zum Alter: Paragondolella ? trammeri nach Original-Beschreibung kommt in der curionii-Zone (Oberfassan) und im unteren Longobard der südalpinen Subprovinz der austroalpinen Conodonten-Provinz vor. Stratum typicum ist eine Ammonitenbank mit Eoprotrachyceras curionii und Proarcestes (Kozur \& Mock, 1973, 13), die der obersten curionii-Zone angehört. In der Tafel-Beschreibung von $G$. trammeri emend. (Kovács \& Kozur, 1980, S. 58, Taf. 6, Fig. 6-8) ist das Alter unteres Longobard, M. hungaricus A.-Z. Nach Krystyn $(1983,239)$ zählt G. trammeri im Epidaurus-Profil zu den wichtigsten Leitformen und hat sich im Grenzbereich Anis/Ladin aus G. eotrammeri entwickelt.

Alter des Rotalgen - Kalkes: Oberfassan, Budurovignathus truempyi A. Z. (von Kozur, 1989, S. 394 aufgestellt).

\section{Astförmige Conodontenelemente}

Paragondolella ? trammeri, der einzige gefundene Plattformconodont in den schwarzen Plattenkalken bei Suhadole, ist von folgenden astförmigen Elementen begleitet: cypridodelliformes Element, enantiognathiformes Element, hindeodelliformes Element, ozarkodiniformes Element und prioniodiniformes Element.

Abb. 5b. Neunfache Vergrösserung des mittleren Abschnittes des dritten Segmentes von Abb. 5 a. In weissen und grauen Zellenlagen sind Lagen mit dicht aneinander stehenden Zellen sichtbar. In schwarzen Feldern ist die Kalzit-Kristallisation erkennbar

Sl. 5b. Devetkratna povečava srednjega dela tretjega segmenta pete slike. V belih in sivih celičnih plasteh so vidne tesno stoječe algine celine. $\mathrm{V}$ črnih poljih je razpoznavna kalcitna kristalizacija 


\section{Andere Mikrofossilreste}

In den Conodontenproben gibt es auch vereinzelte Steinkerne von Foraminiferen und juvenilen Ammoniten. Vereinzelt kommen Reste von Schwebcrinoiden und Fischzähnchen vor. Zahlreich sind Radiolarien- und Spongien-Reste.

\section{Danksagung}

Dr. Ivka Munda, Forschungszentrum der Slowenischen Akademie der Wissenschaften und Kunste danke ich für aufschlussreiche Diskussion und grundlegende Literatur sowie Herrn Prof. Dr. Jost Wendt, Geologisch-Paläontologisches Institut der Universität Tübingen für wichtige Literatur und Herrn Prof. Dr. Robert Riding, Department of Earth Sciences Cardiff, für kritische Bemerkungen.

\section{Schriftum}

Flügel, E. \& Ramovš, A. 1961: Fossilinhalt und Mikrofazies des Dachsteinkalkes (OberTrias) im Begunjščica-Gebirge, S-Karawanken (NW-Slowenien, Jugoslawien. - N. Jb. Geol. Palönt., Mh., 287-294, Stuttgart.

Flügel, E., Ramovš, A. \& Bucur, I.I. 1993: Middle Triassic (Anisian) Limestones from Bled, Northwestern Slovenia: Microfacies and Microfossils. - Geologija 36 (1993), 157-181, Ljubljana.

Johnson, J.H. 1963: Pennsylvanian and Permian algae. - Quart. Colorado School Mines 58, No 3, 211 S., Golden, Colorado.

Kovács, S. \& Kozur, H. 1980: Stratigraphische Reichweite der wichtigsten Conodonten (ohne Zahnreihenconodonten) der Mittel- und Obertrias. - Geol. Paläont. Mitt. Innsbruck 10, 47-78, Innsbruck.

K ozur, H. 1989: Significance of events in conodont evolution for the Permian and Triassic stratigraphy. Courier Forsch.-Inst. Senckenberg 117, 385-408, 1 Fig., 7 Tabs., Frankfurt/M.

Kozur, H. \& Mock, R. 1972: Neue Conodonten aus der Trias der Slowakei und ihre stratigraphische Bedeutung. - Geol. Paläont. Mitt. Innsbruck 2, 1-20, Innsbruck.

Krystyn, L. 1983: Das Epidaurus-Profil (Griechenland) - ein Beitrag zur ConodontenStandardzonierung des tethyalen Ladin und Unterkarn. - Schriftenreihe Erdwiss. Komm., Österr. Akad. Wiss. 5, 231-258, 8 Taf., Wien, New York.

$\mathrm{R}$ a m ov š, A. 1994: Oberfassanische (mitteltriassische) Conodonten aus Kalken südlich von Slugovo, Südslowenien. Geologija 37/38, 141-151, Ljubljana.

Ramovš, A. \& Turnšek, D. 1984: Lower Carnian reef buildups in the northern Julian Alps (Slovenia, NW Yugoslavia). - Razprave IV. razr. SAZU 25, 161-200, 15 Taf., Ljubljana.

Senowbary - Daryan, B. 1981: Zur Paläontologie des Riffes innerhalb der Amphyclinen-Schichten bei Hudajužna, Slowenien. - Razprave IV. razr. SAZU 23, 99-118, 10 Taf., Ljubljana.

Turnšek, D. \& Buser, S. 1989: The Carnian reef complex on the Pokljuka (NW Yugoslavia). - Razprave IV. razr. SAZU 30, 75-127, Ljubljana.

Turnšek, D. \& Ramovš, A. 1987: Upper Triassic (Norian-Rhaetian) reef buildups in the northern Julian Alps (NW Yugoslavia). - Razprave IV. razr. SAZU 28, 27-67, 16 Taf., Ljubljana.

Wend t, J. 1993: Solenoporacean Stromatolites. - Palaios 8, 101-110, Tulsa/OK. 